\title{
ERas regulates cell proliferation and epithelial-mesenchymal transition by affecting Erk/Akt signaling pathway in pancreatic cancer
}

\author{
Yang Liu' ${ }^{1}\left(\mathbb{0} \cdot\right.$ Peng $\mathrm{Qin}^{2} \cdot$ Rong $\mathrm{Wu}^{1} \cdot$ Lianfang $\mathrm{Du}^{1} \cdot \mathrm{Fan} \mathrm{Li}^{1}$
}

Received: 17 January 2020 / Accepted: 14 July 2020 / Published online: 22 July 2020

(c) The Author(s) 2020

\begin{abstract}
Pancreatic cancer is the fourth most common lethal malignancy with an overall 5-year survival rate of less than 5\%. ERas, a novel Ras family member, was first identified in murine embryonic stem cells and is upregulated in various cancers. However, the expression and potential role of ERas in pancreatic cancer have not been investigated. In this study, we found that ERas mRNA and protein were upregulated in pancreatic cancer tissues and cells compared with controls. Knockdown of ERas in pancreatic cancer cells by siRNA significantly decreased cell proliferation, colony formation, migration, and invasion and promoted cell apoptosis in vitro. Epithelial-mesenchymal transition (EMT) is closely related to tumor progression. We observed a significant decrease in $\mathrm{N}$-cadherin expression in pancreatic cancer cells in response to ERas gene silencing by immunofluorescence assay and western blot. Furthermore, tumor growth and EMT were inhibited in xenografts derived from pancreatic cancer cells with ERas downregulation. We further investigated the regulatory mechanisms of ERas in pancreatic cancer and found that ERas may activate the Erk/Akt signaling pathway. Moreover, Erk inhibitor decreased pancreatic cancer cells proliferation and colony formation activities. Our data suggest that targeting ERas and its relevant signaling pathways might represent a novel therapeutic approach for the treatment of pancreatic cancer.
\end{abstract}

Keywords ERas $\cdot$ siRNA $\cdot$ Erk/Akt $\cdot$ Epithelial-mesenchymal transition (EMT) $\cdot$ Pancreatic cancer

\section{Introduction}

Pancreatic cancer is the fourth leading cause of cancerrelated mortalities with rapid progression and dismal prognosis [1-5]. The median survival rate of pancreatic cancer is less than 6 months [6-8]. Chemotherapy plays an important role in the treatment of pancreatic cancer. However, pancreatic cancer can show intrinsic resistance to chemotherapy, resulting in aggressive local invasion and early metastasis

Electronic supplementary material The online version of this article (https://doi.org/10.1007/s13577-020-00401-2) contains supplementary material, which is available to authorized users.

Lianfang Du

shdulf@163.com

Fan Li

medicineli@163.com

1 Department of Ultrasound, Shanghai General Hospital, Shanghai Jiaotong University School of Medicine, Shanghai 200080, China

2 Department of Instrument Science and Engineering, Shanghai Jiao Tong University, Shanghai 200240, China of pancreatic cancer cells (PCCs) $[9,10]$. Thus, there is a desperate need for innovative treatment strategies to improve the prognosis of patients with pancreatic cancer [11].

Embryonic stem (ES) cells are pluripotent cells that maintain differentiation ability [12]. Due to their rapid growth and immortality, ES cells have been used in stem cell therapies. However, ES cells will produce teratomas within several weeks after transplantation, limiting their therapeutic usage [13] Thus, ES cells and tumor cells most likely share common growth properties.

ERas is a new member of the Ras family and shares high identity to conventional Ras oncogenes (Hras, Kras and Nras). The gene encoding ERas, located at chromosome Xp11.23, was first found in murine ES cells [14] and initially identified as a pseudogene $[15,16]$. Subsequent studies showed that ERas plays an important function in the survival of murine ES cells [17-19]. Recent studies have demonstrated that ERas plays a critical role in the occurrence and progression of many malignant tumors, such as gastric cancer [20], breast cancer [21], and neuroblastoma [22].

In this study, we not only identified the expression of ERas in pancreatic cancer cells, but also elucidated the role 
and potential mechanisms of ERas in pancreatic cancer. Meanwhile, we assumed that Erk/Akt pathway helped the development of $\mathrm{PaC}$ which might provide a potential oncogenic mechanism of ERas in PaC. Therefore, early therapy targeting Akt and Erk pathways might produce an inhibitory effect on $\mathrm{PaC}$ and reduce the chemotherapy resistance. Accordingly, we believe ERas might be an extremely key factor participated in cell differentiation and movement via Erk and Akt signaling pathway.

\section{Materials and methods}

\section{Experimental animals}

This study was performed in strict accordance with institutional guidelines and approved by the Institutional Animal Care and Use Committee (IACUC) of the Shanghai Model Organisms Center (permit number 2019-0011). Animals used in this experiment were purchased from Shanghai Slack Laboratory Animals Co., Ltd (China).

\section{Cell lines and reagents}

Human PCC lines BxPC3, Capan-1, CFPAC-1, Panc-1 and SW1990 as well as the normal human pancreatic duct epithelial (HPDE) cell line were obtained from the Type Culture Collection of the Chinese Academy of Sciences. Fetal bovine serum (FBS) was purchased from Gibco BRL (Gaithersburg, MD, USA), and Dulbecco's modified Eagle's medium (DMEM) and RPMI 1640 were obtained from Hyclone (Logan, UT, USA). HPDE, Panc-1 and Capan-1 cells were maintained in DMEM with 10\% FBS. BxPC3 and SW1990 cell lines were maintained in RPMI 1640 with $10 \%$ FBS. CFPAC-1 cells were maintained in Iscove's Modified Dulbecco's Medium (IMDM) with 10\% FBS. All cells were cultured according to standard protocol. The Erk inhibitor SCH772984 was obtained from Selleck Chemicals (Houston, TX, USA) and dissolved in $100 \%$ dimethyl sulfoxide (DMSO) at a concentration of $10 \mathrm{mM}$.

\section{Small interfering RNA transfection}

Two ERas stealth siRNAs (siRNA30 and siRNA32) and the control siRNA were designed and synthesized by Bioneer (Daejon, Korea) (Table 1). siRNAs were mixed with Lipofectamine $^{\mathrm{TM}} 2000$ in Opti-MEM ${ }^{\mathrm{TM}}$ reduced serum medium (31985070, Gibco BRL, Gaithersburg, Md) for $5 \mathrm{~min}$ at room temperature and then added to each well of a 24-well plate containing SW1990 or Panc-1 cells. The cells were harvested for subsequent experiments after $24 \mathrm{~h}$ of transfection.
Table 1 The sequences of ERas siRNA

\begin{tabular}{ll}
\hline Name & Sequences \\
\hline siRNA 30 & F: GCAACUAGCUUUGAGGGAC(dTdT) \\
& R: GUCCCUCAAAGCUAGUUGC(dTdT) \\
siRNA 32 & F: GUAACAUGGGAGUGCCUAA(dTdT) \\
& R: UUAGGCACUCCCAUGUUAC(dTdT) \\
Negative control & F: CCUACGCCACCAAUUUCGU(dTdT) \\
siRNA & R: ACGAAAUUGGUGGCGUAGG (dTd) \\
\hline
\end{tabular}

Table 2 The sequences of primers

\begin{tabular}{ll}
\hline Name & Sequences \\
\hline GAPDH & F: 5'-ggacctgacctgccgtctag-3' \\
& R: 5'-gtagcccaggatgccttga-3' \\
ERas & F: 5'-ggacctgacctgccgtctag-3' \\
& R: 5'-gtagcccaggatgccttga-3' \\
\hline
\end{tabular}

\section{RNA isolation and quantitative real-time PCR (qRT-PCR)}

Total RNA was extracted from cells and tissue samples with Trizol. Reverse transcription was performed using the Takara Reverse Transcription Kit according to the manufacturer's instructions, followed by real-time quantitative PCR using the Takara SYBR Premix ExTaq kit, according to the manufacturer's instructions. The primers are shown in Table 2.

\section{Western blot analysis}

Cells were lysed with RIPA lysis buffer and phenylmethanesulfonyl fluoride (100:1), and protein concentration was determined using the BCA Protein Assay Kit (\#23227, Pierce, USA). Protein samples $(50 \mu \mathrm{g})$ were loaded onto acrylamide gels for electrophoresis and then transferred onto polyvinylidene fluoride membranes. The membranes were then blocked with 5\% non-fat milk for $1 \mathrm{~h}$ at room temperature, followed by incubation with primary antibody overnight at $4{ }^{\circ} \mathrm{C}$. Membranes were then incubated with secondary antibodies for $1 \mathrm{~h}$ at room temperature and the signals were detected by an enhanced chemiluminescence detection system (Amersham Bioscience, Piscataway, NJ, USA). $\beta$-actin was used as the internal control.

The primary antibodies were anti-ERas antibody (1:1000; Abgent), anti-E-cadherin antibody (1:1000; \#14472; Cell Signaling Technology), anti-N-cadherin antibody (1:1000; \#13116; Cell Signaling Technology), anti-Erk (1:1000; \#4695; Cell Signaling Technology), antiphospho-Erk $^{\text {Thr202/Tyr204 }}$ (1:1000; \#9101; Cell Signaling 
Technology), anti-Akt (1:1000; \#4691; Cell Signaling Technology), anti-phospho-Akt ${ }^{\mathrm{Ser} 473}$ (1:1000; \#4060; Cell Signaling Technology), and rabbit anti- $\beta$-actin (1:5000; Abcam). Secondary antibodies included goat anti-rabbit IgG horseradish peroxidase-conjugated secondary antibody (1:2000; \#7074; Cell Signaling Technology) or horse anti-mouse IgG horseradish peroxidase-linked secondary antibody (1:2,000; \#7076; Cell Signaling Technology).

\section{Cell proliferation assays}

Cell proliferation was assessed by CCK- 8 assay and colony formation assay. For CCK-8 assay, siRNA-transfected SW1990 and Panc-1 cells were seeded into 96-well plates $\left(3 \times 10^{3}\right.$ cells/well). After $0,24,48,72$, and $96 \mathrm{~h}$, the absorbance was measured using the Cell Counting Kit (Dojindo, Tokyo, Japan) according to the manufacturer's protocol. For the colony formation assay, siRNA-transfected SW1990 and Panc- 1 cells were seeded in six-well plates (200 cells/ well) and cultured for 14 days. Colonies were fixed with $1 \mathrm{ml} 4 \%$ paraformaldehyde containing $0.04 \%$ crystal violet for $20 \mathrm{~min}$. The supernatant was discarded and cells were washed with $1 \mathrm{ml} \mathrm{ddH_{2 }} \mathrm{O}$. Images were obtained in five random fields and colonies were counted by ImageJ software. Each experiment was replicated with five independent wells.

\section{Apoptosis assay}

Flow cytometry was used to determine the percentage of apoptotic cells. Cells were double-labeled with Annexin $\mathrm{V}$ (AV)-fluorescein isothiocyanate (FITC) and propidium iodide (PI). Cells were washed twice and adjusted to a concentration of $1 \times 10^{6}$ cells $/ \mathrm{mL}$ with cold PBS. Binding buffer $(195 \mu \mathrm{l})$, AV-FITC $(5 \mu \mathrm{l})$ and PI $(10 \mu \mathrm{l})$ were added to 100 $\mu \mathrm{L}$ of cell suspension and samples were incubated for $20 \mathrm{~min}$ at room temperature in the dark. Samples were examined on a Beckman Coulter Flow Cytometer. The data were analyzed by FlowJo 10.

\section{Hoechst 33342 assay}

Hoechst 33,342 staining was used to evaluate cell apoptosis. Cells transfected with siRNA were incubated with Hoechst 33342 (Beyotime, Nantong, China) at $37^{\circ} \mathrm{C}$ with $5 \% \mathrm{CO}_{2}$ for $20 \mathrm{~min}$ according to the manufacturer's protocol. Apoptosis was then assessed by fluorescence microscopy based on the presence of condensed chromatin and micronucleation.

\section{Cell migration and invasion assay}

Cell migration and invasion abilities were assessed by Transwell assay (Corning, California, USA) according to the manufacturer's instructions. In migration assays,
siRNA-transfected SW1990 $\left(3 \times 10^{4}\right)$ and Panc-1 cells $\left(3 \times 10^{4}\right)$ were resuspended in $200 \mu 1$ serum-free medium and seeded into the upper chamber of the Transwell system. In the invasion assay, Matrigel (BD Biosciences) was included to simulate the extracellular matrix; siRNA-transfected SW1990 $\left(5 \times 10^{4}\right)$ and Panc- 1 cells $\left(5 \times 10^{4}\right)$ resuspended in $200 \mu \mathrm{l}$ serum-free medium were seeded into the upper chamber pre-coated with Matrigel. In both migration and invasion assays, the lower chambers were filled with $500 \mu \mathrm{l}$ of medium containing $20 \% \mathrm{FBS}$ as the chemoattractant. Cells were incubated for another $48 \mathrm{~h}$ at $37{ }^{\circ} \mathrm{C}$ with $5 \%$ $\mathrm{CO}_{2}$ and then the non-migrating cells on the upper surface of the chamber were gently scraped off. The cells were fixed with $4 \%$ paraformaldehyde containing $0.04 \%$ crystal violet for $20 \mathrm{~min}$. Migrated or invaded cells were counted under a microscope in at least three random fields.

\section{Wound-healing assay}

SW1990, siRNA-transfected SW1990, Panc-1 and siRNAtransfected Panc-1 cells were seeded into six-well plates and cultured until they achieved $90 \%$ confluence. The cell monolayers were then scratched with $200 \mu$ l pipette tips. Cells were photographed at 0 and $24 \mathrm{~h}$ using an inverted microscope, and the wound width was measured by Image $\mathbf{J}$ software. Each analysis was repeated five times.

\section{Colony formation assay}

A total of 200 siRNA-transfected SW1990 or SW1990 cells incubated with DMSO and Erk inhibitor (SCH772984) were plated in six-well plates for 2 weeks. Cell colonies were fixed with $4 \%$ methanol for $20 \mathrm{~min}$ and stained with $0.04 \%$ crystal violet for $20 \mathrm{~min}$. After washing with PBS for $10 \mathrm{~min}$ and air drying, colonies were photographed in five random fields and counted by ImageJ software.

\section{Immunofluorescence assay}

EMT, both in vitro and in vivo, was analyzed by immunofluorescence. SW1990 cells were fixed in 4\% paraformaldehyde for $20 \mathrm{~min}$ and then permeabilized with $0.1 \%$ Triton $\mathrm{X}-100$ for 15 min and then incubated with $5 \%$ bovine serum albumin (BSA) for $30 \mathrm{~min}$. While in vivo analysis, dewaxed and hydrated mice tumor sections were heated to $95-100{ }^{\circ} \mathrm{C}$ in sodium citrate solutions for $30 \mathrm{~min}$ in order to repair the antigens. Afterward, sections were also incubated with 5\% BSA at room temperature. Both cell samples and tumor sections were incubated with anti-E-cadherin antibody and anti-N-cadherin antibody $(1: 100)$ overnight at $4{ }^{\circ} \mathrm{C}$ and then incubated with FITC-conjugated goat anti-mouse/rabbit secondary antibodies (1:2000; \#SA00003-1/\#SA00003-2; 
Proteintech) at room temperature for $1 \mathrm{~h}$. An Olympus BX-43 microscope was used to capture images.

\section{Tumor xenograft model and tumorigenicity assay}

Twelve 4-week-old male nude mice (weight, approximately $20 \mathrm{~g}$ ) were housed under specific pathogen-free conditions. Mice were randomly divided into three groups (control, siRNA30 and siRNA32 groups; $n=4$ mice/group). siRNAtransfected SW1990 cells $\left(3 \times 10^{6}\right.$ cells in $100 \mu$ l of PBS) were subcutaneously implanted into the back of mice. The tumor nodules were examined weekly with a caliper. Tumor volume was calculated by the following formula: volume $=0.5 \times$ length $\times$ width $^{2}$. Tumor growth curves were calculated. The nude mice were euthanized when the volume of tumors reached $1 \mathrm{~cm}^{3}$ in size (after approximately 4 weeks). Tumors were harvested, dissected, weighed and embedded in paraffin or fixed in $10 \%$ formalin.

\section{Immunohistochemistry}

Tissues $(4 \mu \mathrm{m}$ thick) were stained with hematoxylin and eosin (H\&E). The FITC Rabbit anti-Ki-67 antibody (1:100, 12075, CST) was used to detect Ki-67 expression (nuclear staining), and the Ki-67 index was determined as the percentage of Ki-67-positive cells in 100 cells. The TUNEL assay (Roche, Germany) was performed to detect apoptotic cells in tumor tissues. An Olympus BX-43 microscope was used to capture images.

\section{Statistical analysis}

Statistical analyses were performed using SPSS 16.0 software. Statistical comparisons were conducted by Student's $t$ test and the results are presented as mean \pm standard

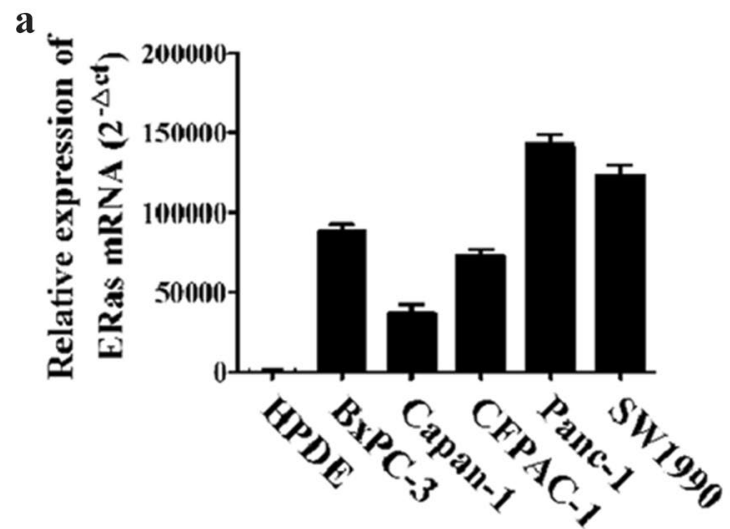

Fig. 1 ERas mRNA and protein expression in PCCs. a Relative ERas gene expression was examined in five pancreatic cancer cell lines and the normal human pancreatic duct epithelial cell HPDE by real-time deviation (SD) from at least three separate experiments. A $P$ value of 0.05 or less was considered statistically significant.

\section{Results}

\section{ERas expression is significantly upregulated in pancreatic cancer}

To determine whether ERas is involved in the development of pancreatic cancer, we first analyzed ERas mRNA and protein expression in HPDE and pancreatic cancer cell lines by real-time PCR and western blot analysis. Both ERas mRNA and protein expression were detected in all five pancreatic cancer cell lines, while no expression was detected in the HPDE cell line (Fig. 1a, b). Taken together, these results showed that ERas mRNA and protein expression are significantly upregulated in PCCs.

\section{Inhibition of ERas expression decreased the proliferation and colony formation abilities of PCCs in vitro}

We next examined the effect of ERas on pancreatic cancer pathogenesis. Our results showed that both SW1990 and Panc- 1 exhibit high endogenous expression of ERas, and thus SW1990 and Panc-1 were used for subsequent functional assays. We downregulated the expression of ERas in the cell lines using two siRNAs (siRNA30 and siRNA32) and confirmed that the expression levels of ERas were markedly decreased in both SW1990 and Panc-1 cells after siRNA30 and siRNA32 transfection compared with cells transfected with control siRNA (Fig. S1). We next examined the role of ERas in PCCs proliferation. As shown in Fig. 2a, b, CCK-8 assays revealed a significant reduction

b

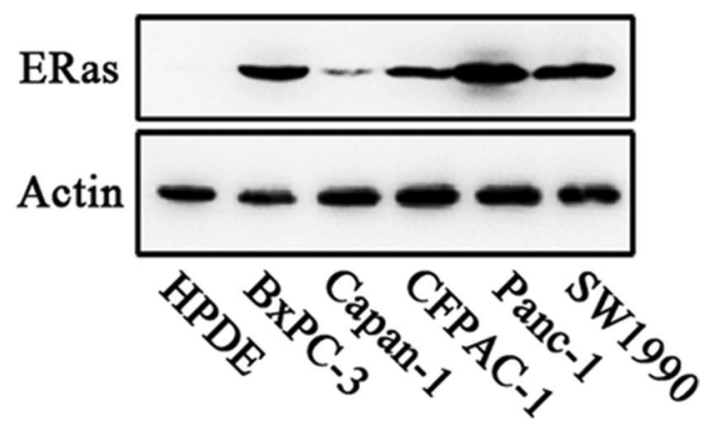

PCR. b Expression of ERas protein in the indicated cell lines was determined by western blotting 

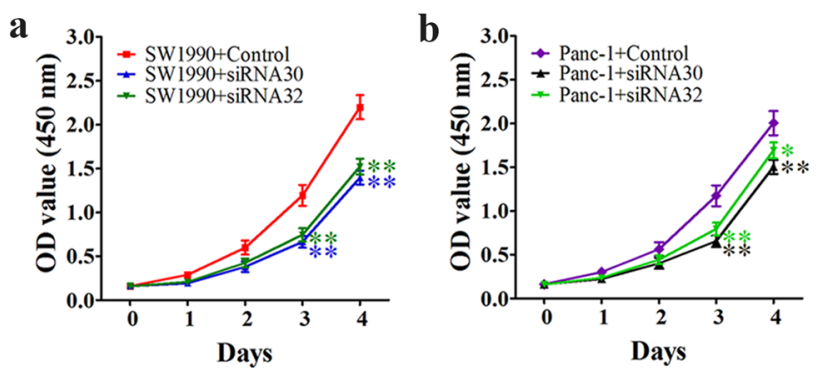

c
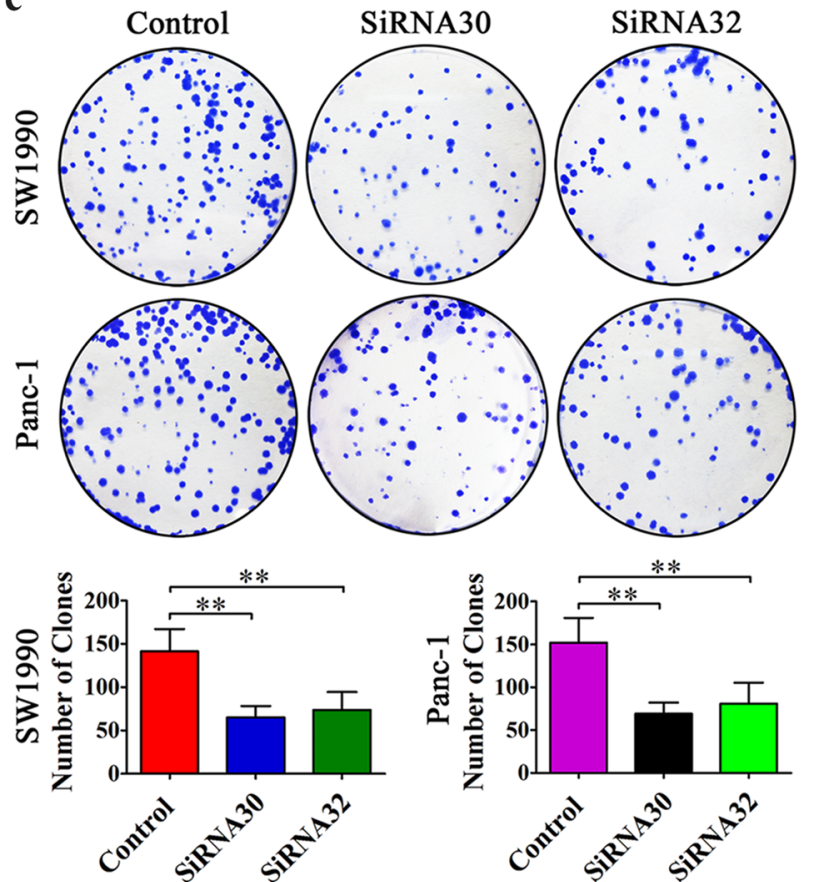

Fig. 2 The role of ERas in PCCs proliferation and apoptosis in vitro. a, b SW1990 and Panc-1 cells transfected with ERas siRNA30, ERas siRNA32 or control siRNA were plated at $3 \times 10^{3}$ cells/well in 96-well plates. CCK-8 assays were performed at 0, 24, 48, 72 and $96 \mathrm{~h}$. The experiment was repeated three times and data are shown as mean $\pm \mathrm{SD}(* P<0.05$ and $* * P<0.01$ vs. control). c After $24 \mathrm{~h}$ of siRNA30, siRNA32 and control transfection, $2 \times 10^{2}$ cells were transferred into 6-well plates and plates were incubated for 2 weeks. Colonies were stained with crystal violet and photographed by an ordinary camera. Bar charts (bottom) show the number of colonies. The experiment was repeated three times and the significance was analyzed using the Student's $t$ test. Data are shown as mean \pm SD

of growth in both cell lines silenced for ERas gene expression compared with control cells. We also found that inhibition of ERas resulted in fewer and smaller colonies than the control groups in colony formation assays (Fig. 2c). These results demonstrate that downregulation of ERas significantly inhibited the proliferation and colony formation ability of PCCs.
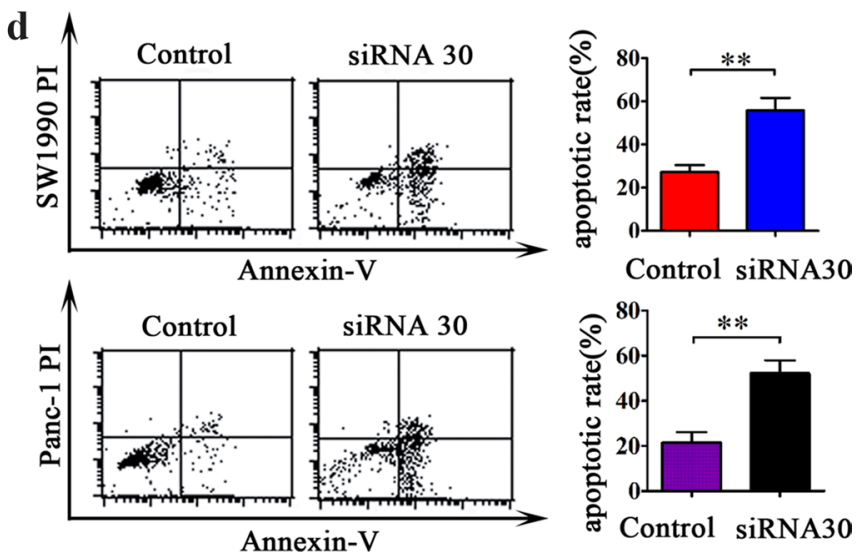

$\mathbf{e}$
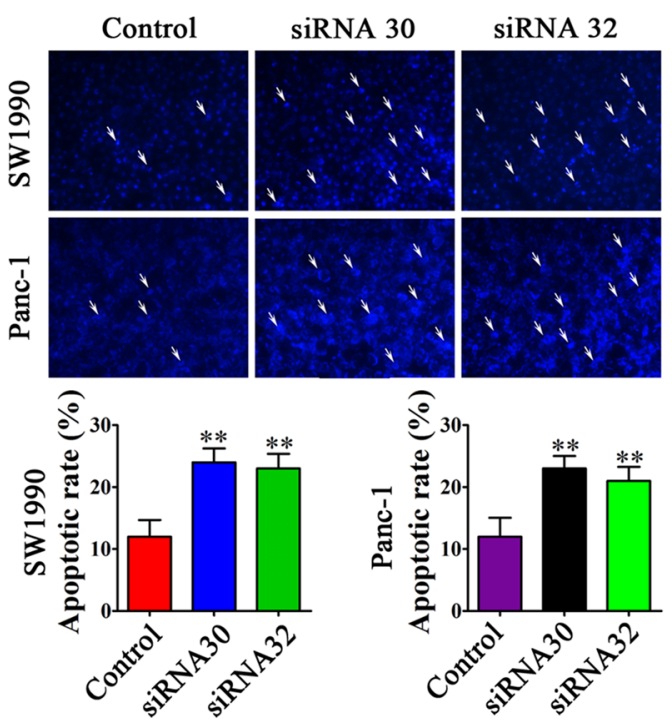

(*P<0.05 and $* * P<0.01$ vs. control). d Apoptosis was evaluated in SW1990 and Panc-1 cells transfected with siRNA30 or control siRNA by Annexin V-PI staining and flow cytometry. The experiment was repeated three times and the representative histograms are shown. The apoptotic rates are shown as mean \pm SD $(* P<0.05$ and $* * P<0.01$ vs. control). e Apoptosis was evaluated in SW1990 and Panc-1 cells transfected as indicated and stained with Hoechst 33342 staining (original magnification $\times 200$ ). The apoptotic rates of SW1990 and Panc-1 cells in five random fields were counted and the experiment was repeated three times independently. Data are shown as mean $\pm \mathrm{SD}(* * P<0.01$ vs. control)

\section{Downregulation of ERas promoted the apoptosis of PCCs}

We further examined the effects of ERas on apoptosis in vitro using flow cytometry and Hoechst 33342. Flow cytometry assays showed that the apoptotic rate was significantly increased in ERas siRNA30-transfected 

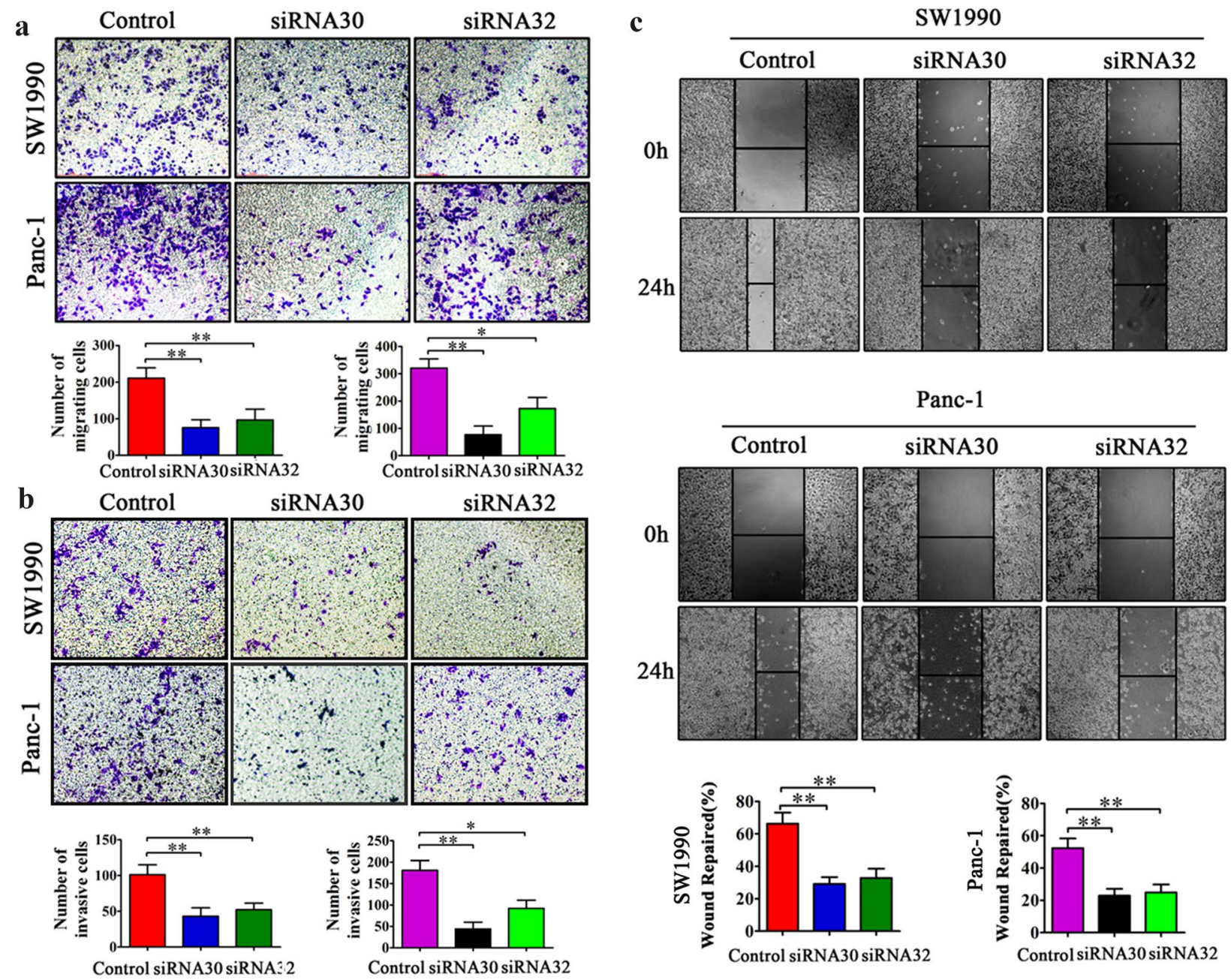

siRNA30 SiRNA32

d
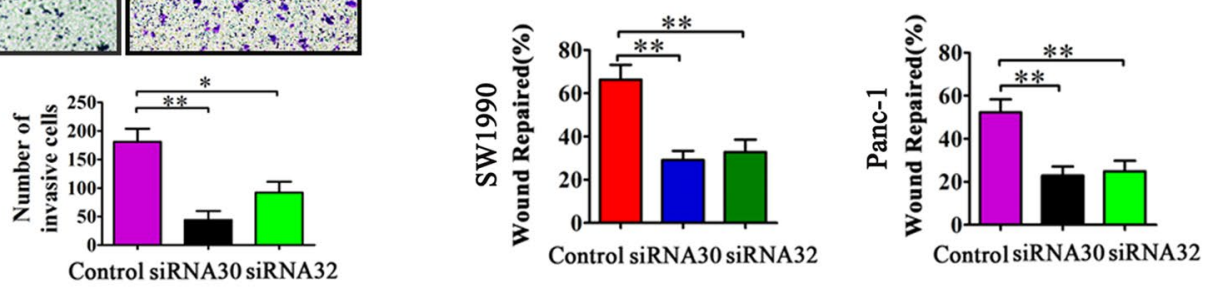

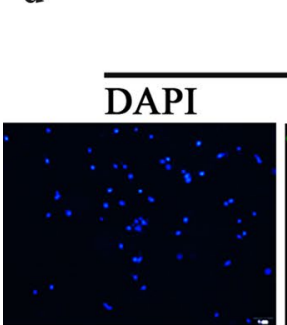

DAPI

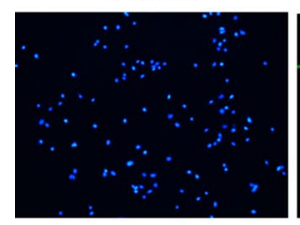

\section{Control} E-cadherin

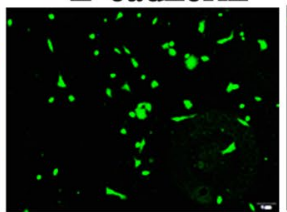

$\mathrm{N}$-cadherin

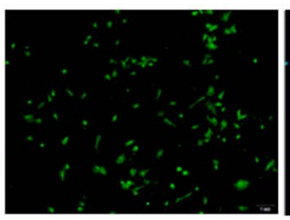

Merge

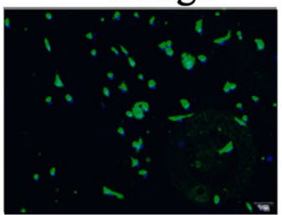

Merge

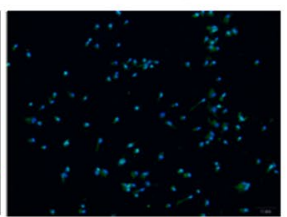

SW1990

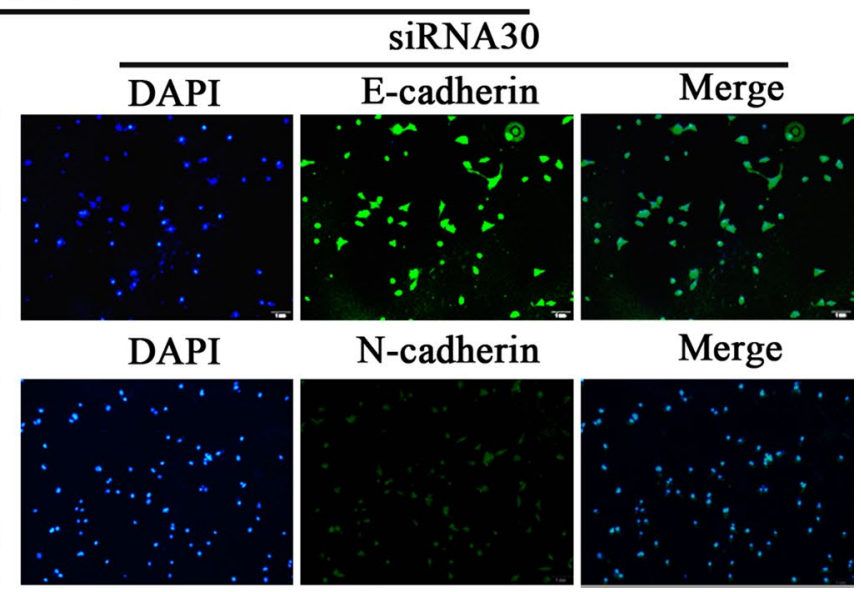

cells compared with control cells $(55.78 \% \pm 6.21 \%$ vs. $28.21 \% \pm 3.89 \%$ in SW $1990,52.19 \% \pm 5.77 \%$ vs. $21.34 \% \pm 4.85 \%$ in Panc-1, respectively; $P<0.01$ ) (Fig. 2d). ERas siRNA-transfected cells exhibited the typical morphologic features of apoptosis, such as condensed chromatin and DNA fragments, as shown by Hoechst
33,342 staining (Fig. 2e). We observed more condensed and fragmented nuclei in ERas siRNA-treated cells compared with the control groups $(P<0.01)$. 
4Fig. 3 The role of ERas in SW1990 and Panc-1 cell migration. a After $24 \mathrm{~h}$ of siRNA30, siRNA32 and control siRNA transfection, $3 \times 10^{4}$ cells were transferred to Transwell chambers and incubated for another $48 \mathrm{~h}$. Cells were stained with crystal violet and observed by microscopy ( $\times 50$ magnification; Zeiss). The number of migrating cells in five random fields was counted using ImageJ software $(\times 100$ magnification; Zeiss). The experiment was repeated three times and data are shown as mean $\pm \mathrm{SD}(* P<0.05, * * P<0.01$ vs. control). b siRNA-transfected SW1990 $\left(5 \times 10^{4}\right)$ and Panc- 1 cells $\left(5 \times 10^{4}\right)$ were resuspended in $200 \mu \mathrm{l}$ serum-free medium and seeded into the upper chamber of Transwell chambers pre-coated with Matrigel. Cells were incubated for another $48 \mathrm{~h}$ and fixed with $4 \%$ paraformaldehyde containing $0.04 \%$ crystal violet for $20 \mathrm{~min}$. Invasive cells were imaged and counted under a microscope in five random fields $(\times 100$ magnification; Zeiss); The experiment was repeated three times and data are shown as mean $\pm \mathrm{SD}(* P<0.01$, $* * P<0.01$ vs. control). c Migration activity was also measured by wound-healing assay. Migration distance was measured from five random fields captured at each indicated time point. The experiment was repeated three times and wound repair percentage of each cell line is shown in bar charts as mean \pm SD $(* * P<0.01$ vs. control). (D) SW1990 transfected as indicated were analyzed by immunofluorescence using the indicated antibodies and captured images by Olympus BX-43 microscope. The experiment was repeated three times and the representative pictures are shown

\section{ERas promoted PCC migration and invasion in vitro}

We next used Transwell assay and wound-healing assay to ascertain the role of ERas in the migration and invasion of PCCs. The results showed that downregulation of ERas gene significantly inhibited SW1990 and Panc-1 cell migration and invasion compared with controls (Fig. 3a-c). These results suggest that ERas may play a key role in the metastasis of PCCs. As shown in Fig. 3d, the expression level of the mesenchymal cell marker $\mathrm{N}$-cadherin decreased and the epithelial cell marker E-cadherin increased when ERas was downregulated by siRNA. Taken together, these data suggest that ERas promotes pancreatic cancer cell migration, invasion, and EMT.

\section{ERas promoted tumorigenicity and EMT of pancreatic cancer in vivo}

We then explored the role of ERas in pancreatic cancer tumor progression in vivo. ERas downregulated SW1990 cells or control siRNA-treated SW1990 cells were subcutaneously injected into nude mice. After 4 weeks, the mice were killed, and the xenograft tumors were extracted (Fig. 4a). Decreased tumor volumes and weights were found in the ERas downregulation group compared with the control group (Fig. 4b). The expression of E-cadherin, $\mathrm{N}$-cadherin and $\beta$-actin were determined by western blotting in siRNA-transfected subcutaneous tumors. We observed that $\mathrm{N}$-cadherin expression was decreased and E-cadherin expression was increased in the siRNA30 and siRNA32 groups compared with controls (Fig. 4c). H\&E staining showed the morphology of xenotransplanted tumor (Fig. 4d). Immunohistochemical staining revealed significantly fewer Ki-67-positive proliferative cells in xenograft tumors derived from cells transfected with ERas siRNA30 than in the control group $(P<0.01)$ (Fig. 4e). We also performed TUNEL assays to investigate the effects of ERas on cell apoptosis in vivo and observed that downregulation of ERas resulted in increased apoptosis in xenograft tumors of SW1990 cells $(P<0.01)$ (Fig. 4f). Besides, immunofluorescence staining also showed that $\mathrm{N}$-cadherin expression was decreased and E-cadherin expression was increased in ERas downregulation group (Fig. 4g). Together these data indicate that ERas promotes pancreatic cancer tumor formation and EMT in vivo. Thus, ERas may act as a novel tumor-promoting factor and play a critical role in pancreatic cancer development.

\section{Regulation of PCCs activity by ERas is significantly associated with Erk/Akt signaling pathways}

To ascertain the molecular mechanisms by which ERas promotes pancreatic cancer progression, we examined several signaling transduction pathways that might be crucial in tumorigenesis and Erk and Akt were focused on in the following experiments. As shown in Fig. 5a, the expression of phosphorylated Erk ${ }^{\mathrm{Thr} 202 / \mathrm{Tyr} 204}$ and $\mathrm{Akt}^{\mathrm{Ser} 473}$ were reduced in the ERas-silenced cells compared with the control groups. We thus examined the role of Erk in pancreatic cell function using the Erk inhibitor SCH772984 and found the proliferation and colony formation of PCCs were inhibited by SCH772984 (Fig. 5b, c). These results suggest that ERas may regulate the proliferation, migration and colony formation of PCCs by regulating Erk-Akt signaling (Fig. 5d). These data also strongly suggest that Erk-Akt signaling is significantly associated with pancreatic cancer progression.

\section{Discussion}

Pancreatic cancer is the most lethal malignancy in humans with aggressive local invasion and early metastasis [23,24] and an incidence that has increased over the last decades [25]. The Ras family plays a critical role in carcinogenesis and the development of pancreatic cancer. Growing evidence has supported the critical function of the Ras gene on the pathologic states of pancreatic cancer [26]. However, the expression and function of ERas, a member of the Ras family, in pancreatic cancer have been unknown.

ERas, previously named Hrasp, is located on the $\mathrm{X}$ chromosome and has a single open reading frame encoding a polypeptide with $76 \%$ identity to mouse ERas [20]. Previous studies have shown that ERas is pivotal in the occurrence and development of various malignant tumors, 

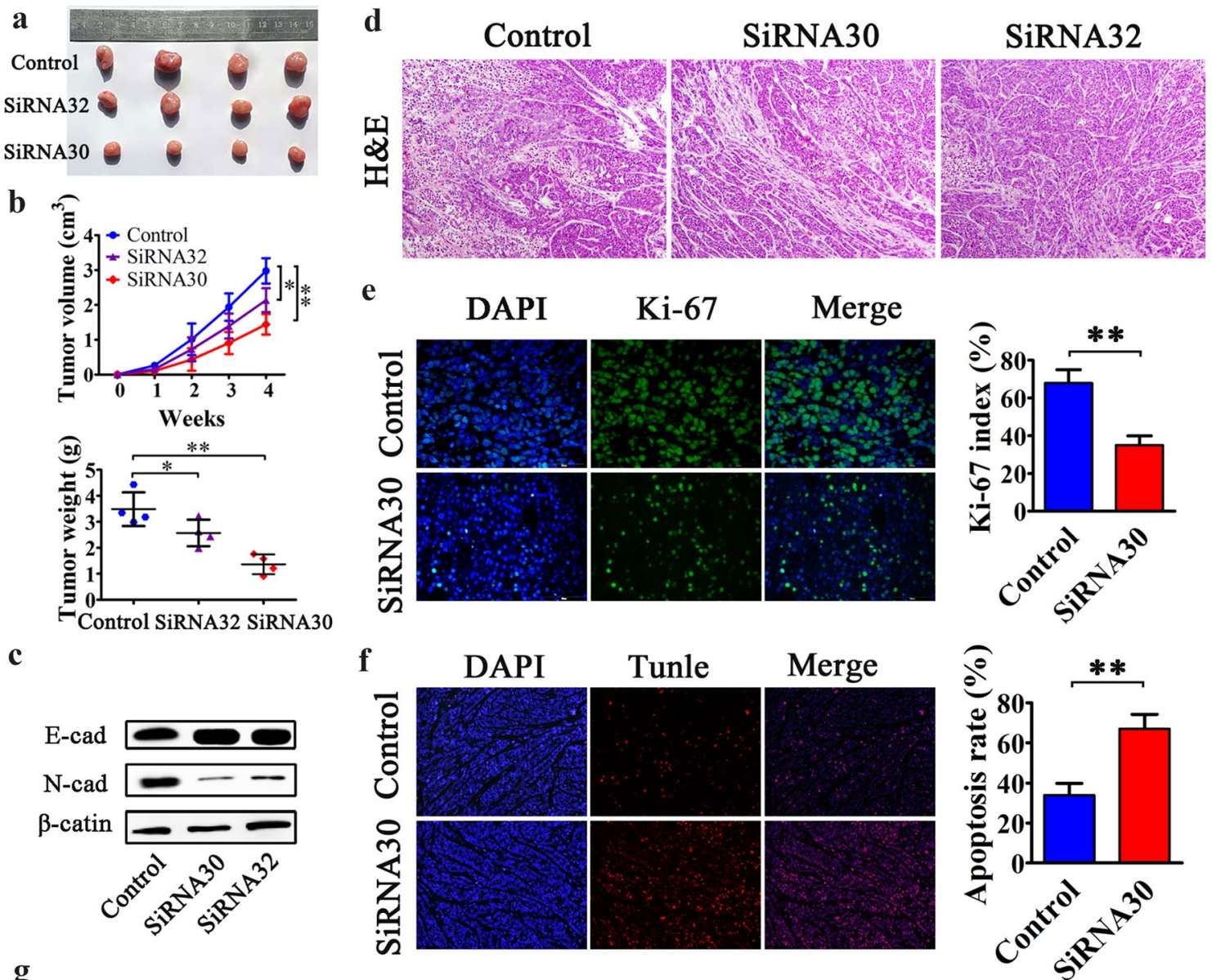

g
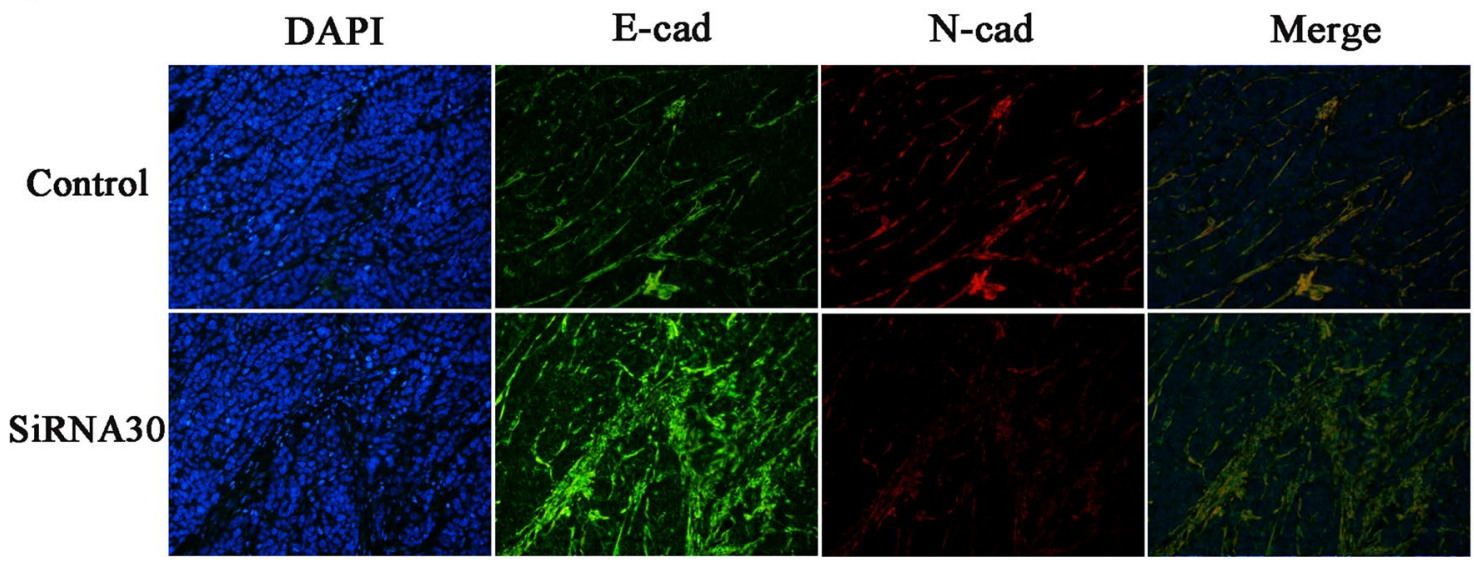

Fig. 4 ERas promotes tumorigenicity in vivo and activates Erk/Akt signaling to regulate the activity of PCCs. a Control siRNA-, ERas siRNA30- and siRNA32-transfected SW1990 cells were injected into the right side of nude mice ( $n=4 /$ group). The mice were sacrificed after 4 weeks. ERas siRNA-transfected SW1990 cells exhibited slower growth compared with controls. b Reduced tumor volumes and weights were observed in xenografts derived from ERas siRNA-transfected SW1990 cells $(* P<0.01, * * P<0.01$ vs. control). c SW1990 cells transfected with control siRNA, ERas siRNA30 and siRNA32 were harvested and E-cadherin, $\mathrm{N}$-cadherin and $\beta$-actin expressions were determined by western blotting. d HE staining revealed the morphology of xenografts from the indicated groups. $\mathbf{e}$
Immunohistochemical staining showed that xenograft tumors derived from ERas siRNA30-expressing cells contained significantly fewer Ki-67-positive cells than those from the control group. The Ki-67 index was determined as the percentage of $\mathrm{Ki}-67$-positive cells in 100 cells and the experiment was repeated three times independently. Data are shown in bar charts as mean $\pm \mathrm{SD}(* * P<0.01$, siRNA30 vs. control). f ERas significantly inhibited apoptosis in xenograft tumors of SW1990 cells as shown by TUNEL assay. The apoptosis rate was counted in five random fields and the experiment was repeated three times. Data are shown as mean \pm SD $(* * P<0.01$, siRNA30 vs. control). $g$ The expression of E-cadherin and $\mathrm{N}$-cadherin in xenograft tumors were analyzed by immunofluorescence 
a

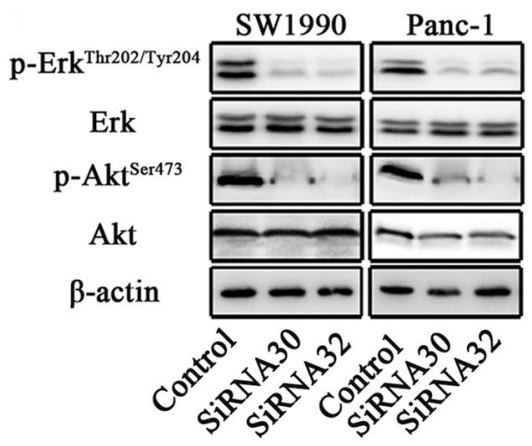

c

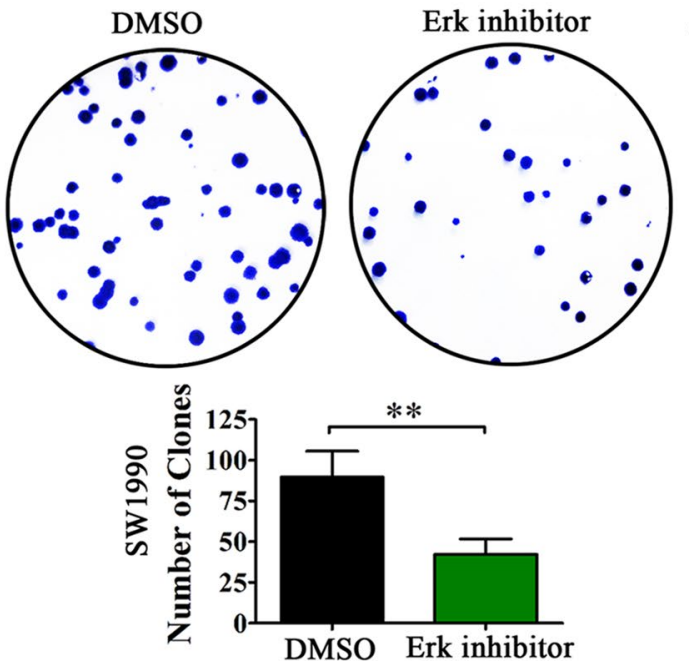

b

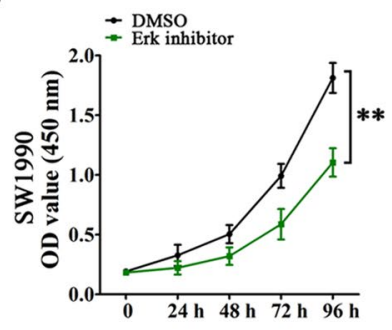

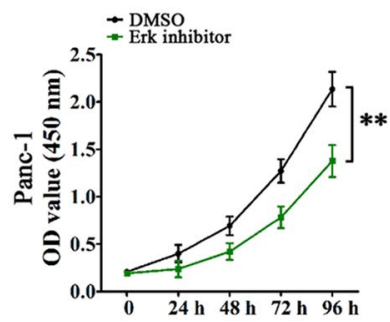

d

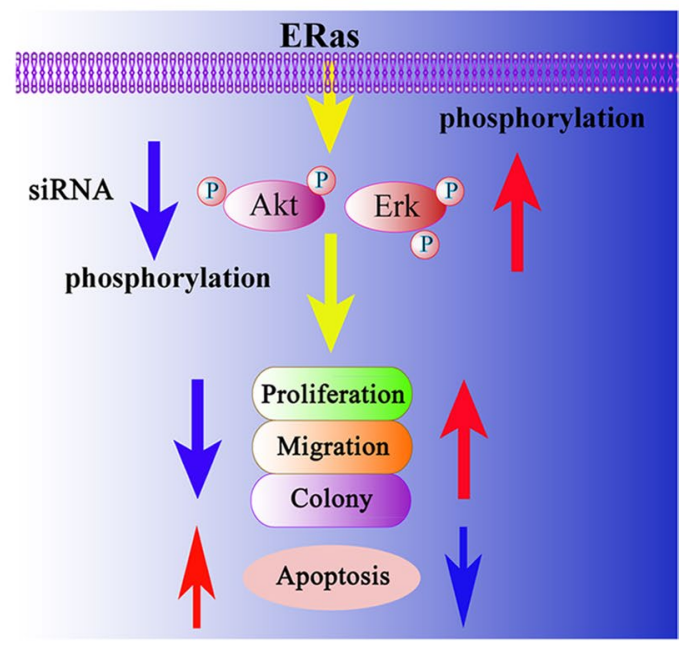

Fig. 5 ERas activates Erk/Akt signaling to regulate the activity of PCCs. a Immunoblotting of phospho-Erk $\left(\mathrm{Thr}^{202} / \mathrm{Tyr}^{204}\right)$ and phospho-Akt $\left(\mathrm{Ser}^{473}\right)$ levels in SW1990 and Panc-1 cells treated with siRNA. ERas levels were normalized to those of $\beta$-actin. b Cell

especially gastric cancer $[19,20,27]$. A previous study showed that ERas was strongly expressed in 142 gastric cancer tissues and closely related to liver or lymph node metastases [20]. In our study, we revealed that ERas is highly expressed in pancreatic cancer and exerted tumorpromoting effects. CCK-8, colony formation assays and Transwell assays revealed that ERas regulates PCC proliferation, colony formation, migration, invasion and metastasis activities as well as inhibits apoptosis in vitro. Importantly, subcutaneous tumor growth was inhibited in xenografts derived from cells with ERas knockdown. Ki-67 immunohistochemical staining demonstrated significantly fewer proliferative cells in ERas knockdown xenograft tumors, indicating ERas enhances the proliferation of PCCs in tumors. TUNEL assays also showed increased apoptotic cells in ERas knockdown xenograft tumors, suggesting ERas inhibits the apoptosis of tumor cells in vivo. Expression level of the mesenchymal cell marker N-cadherin was also decreased when ERas was downregulated by siRNA in vitro and in vivo. It is worth mentioning that we also found ERas was involve in the distant metastases, growth was inhibited by Erk inhibitor (SCH772984). c Colony formation ability was inhibited by Erk inhibitor (SCH772984) in SW1990 cells. d ERas activates Erk/Akt signaling to regulate the activity of pancreatic cancer cells

suggesting that ERas may serve as a potential diagnostic marker in pancreatic cancer.

Chemotherapy plays an important role in the comprehensive treatment of pancreatic cancer. However, resistance to chemotherapy is a big hurdle to treat this disease [28,29]. Thus, the determining mechanisms of pancreatic cancer may identify new alternate targets for treatment. The Akt pathway plays a critical role in the proliferation and activity of tumor cells. ERas may exert its function in pancreatic cancer by activating and regulating various downstream signaling transduction pathways. We hypothesized that ERas might modulate Erk and Akt signal transduction pathways to induce the cellular condition. Activation of signaling cascades by ERas may significantly enhance pancreatic cancer cells proliferation, migration, and colony formation ability and inhibit apoptosis. We found that inhibition of ERas expression by siRNA reduced the expression of Erk and Akt. In addition, inhibition of the Erk pathway using the Erk inhibitor SCH772984 resulted in greatly decreased proliferation and colony formation of pancreatic cancer cells. Together, these findings suggest that ERas may potentiate 
the activities of pancreatic cancer cells through the Erk and Akt pathway, suggesting that these pathways may represent a suitable target for the therapeutic treatment of pancreatic cancer.

In conclusion, we confirmed that ERas was overexpressed in pancreatic cancer tissues and PCCs. In addition, we revealed the biological role and potential signal pathways of ERas in pancreatic cancer progression. ERas was activated in pancreatic cancer cells, where ERas plays a critical role in PCCs survival and EMT. Our results suggest that chemotherapy strategies using low-dose Erk inhibitor drugs might provide a beneficial therapeutic approach for the clinical application of pancreatic cancer.

Acknowledgements We would like to acknowledge the funding support from the Medical and Engineering Cross-Foundation of Shanghai Jiaotong University (Nos. ZH2018QNA21), Shanghai Municipal Commission of Health and Family Planning (20184Y0363), Songjiang District Science and Technology Research Projects of Shanghai (19SJKJGG29) and the National Natural Science Foundation of China (No. 81571679 and No. 81771838). We also thank Liwen Bianji, Edanz Editing China (www.liwenbianji.cn/ac), for editing the English text of a draft of this manuscript.

Author contributions YL, LD and FL designed the study. YL, PQ and RW performed the experiments and analyzed the data. YL wrote the manuscript in consultation with LD and FL. All authors read and approved the final manuscript.

\section{Compliance with ethical standards}

Conflict of interest The authors declare no competing interests.

Ethical approval This study was performed in strict accordance with institutional guidelines and approved by the Institutional Animal Care and Use Committee (IACUC) of the Shanghai Model Organisms Center (permit number 2019-0011)

Open Access This article is licensed under a Creative Commons Attribution 4.0 International License, which permits use, sharing, adaptation, distribution and reproduction in any medium or format, as long as you give appropriate credit to the original author(s) and the source, provide a link to the Creative Commons licence, and indicate if changes were made. The images or other third party material in this article are included in the article's Creative Commons licence, unless indicated otherwise in a credit line to the material. If material is not included in the article's Creative Commons licence and your intended use is not permitted by statutory regulation or exceeds the permitted use, you will need to obtain permission directly from the copyright holder. To view a copy of this licence, visit http://creativecommons.org/licenses/by/4.0/.

\section{References}

1. Al-Husseini MJ, Saad AM, Turk T, Tabash MA, Abdel-Rahman O. Impact of prior malignancy on survival outcomes of stage IV pancreatic adenocarcinoma: SEER-based cohort. J Gastrointest Cancer. 2019;50(4):794-800.
2. Yu J, Blackford AL, Dal Molin M, Wolfgang CL, Goggins M. Time to progression of pancreatic ductal adenocarcinoma from low-to-high tumour stages. Gut. 2015;64(11):1783-9.

3. Wu J, Li H, Shi M, Zhu Y, Ma Y, Zhong Y, Xiong C, Chen H, Peng C. TET1-mediated DNA hydroxymethylation activates inhibitors of the Wnt/beta-catenin signaling pathway to suppress EMT in pancreatic tumor cells. J Exp Clin Cancer Res. 2019;38(1):348.

4. Sasaki N, Toyoda M, Hasegawa F, Fujiwara M, Gomi F, Ishiwata T. Fetal bovine serum enlarges the size of human pancreatic cancer spheres accompanied by an increase in the expression of cancer stem cell markers. Biochem Biophys Res Commun. 2019;514(1):112-7.

5. Zahir MN, Jabbar AA. Metastatic pancreatic carcinoma and experience with FOLFIRINOX - a cross sectional analysis from a developing country. Asian Pac J Cancer Prev. 2015;16(14):6001-6.

6. McCarroll JA, Naim S, Sharbeen G, Russia N, Lee J, Kavallaris M, Goldstein D, Phillips PA. Role of pancreatic stellate cells in chemoresistance in pancreatic cancer. Front Physiol. 2014;5:141

7. Abate-Daga D, Lagisetty KH, Tran E, Zheng Z, Gattinoni L, Yu Z, Burns WR, Miermont AM, Teper Y, Rudloff U, Restifo NP, Feldman SA, Rosenberg SA, Morgan RA. A novel chimeric antigen receptor against prostate stem cell antigen mediates tumor destruction in a humanized mouse model of pancreatic cancer. Hum Gene Ther. 2014;25(12):1003-122.

8. Yan H, Wu J, Liu W, Zuo Y, Chen S, Zhang S, Zeng M, Huang W. MicroRNA-20a overexpression inhibited proliferation and metastasis of pancreatic carcinoma cells. Hum Gene Ther. 2010;21(12):1723-34.

9. Meng Q, Shi S, Liang C, Liang D, Hua J, Zhang B, Xu J, Yu X. Abrogation of glutathione peroxidase-1 drives EMT and chemoresistance in pancreatic cancer by activating ROS-mediated Akt/ GSK3beta/Snail signaling. Oncogene. 2018;37(44):5843-57.

10. Su D, Yamaguchi K, Tanaka M. The characteristics of disseminated tumor cells in pancreatic cancer: a black box needs to be explored. Pancreatology. 2005;5(4-5):316-24.

11. Vassaux G, Angelova A, Baril P, Midoux P, Rommelaere J, Cordelier P. The promise of gene therapy for pancreatic cancer. Hum Gene Ther. 2016;27(2):127-33.

12. Yasuda K, Yashiro M, Sawada T, Ohira M, Hirakawa K. ERas oncogene expression and epigenetic regulation by histone acetylation in human cancer cells. Anticancer Res. 2007;27(6B):4071-5.

13. Liu Y, Wang Z, Li H, Wu Z, Wei F, Wang H. Role of the ERas gene in gastric cancer cells. Oncol Rep. 2013;30(1):50-6.

14. Takahashi K, Mitsui K, Yamanaka S. Role of ERas in promoting tumour-like properties in mouse embryonic stem cells. Nature. 2003;423(6939):541-5.

15. Chang EH, Gonda MA, Ellis RW, Scolnick EM, Lowy DR. Human genome contains four genes homologous to transforming genes of Harvey and Kirsten murine sarcoma viruses. Proc Natl Acad Sci USA. 1982;79(16):4848-52.

16. Miyoshi J, Kagimoto M, Soeda E, Sakaki Y. The human c-Ha-ras2 is a processed pseudogene inactivated by numerous base substitutions. Nucleic Acids Res. 1984;12(4):1821-8.

17. Kameda T, Thomson JA. Human ERas gene has an upstream premature polyadenylation signal that results in a truncated, noncoding transcript. Stem Cells. 2005;23(10):1535-40.

18. Roperto S, Russo V, Urraro C, Restucci B, Corrado F, De Falco F, Roperto F. ERas is constitutively expressed in full term placenta of pregnant cows. Theriogenology. 2017;103:162-8.

19. Kaizaki R, Yashiro M, Shinto O, Yasuda K, Matsuzaki T, Sawada T, Hirakawa K. Expression of ERas oncogene in gastric carcinoma. Anticancer Res. 2009;29(6):2189-93. 
20. Kubota E, Kataoka H, Aoyama M, Mizoshita T, Mori Y, Shimura T, Tanaka M, Sasaki M, Takahashi S, Asai K, Joh T. Role of ES cell-expressed Ras (ERas) in tumorigenicity of gastric cancer. Am J Pathol. 2010;177(2):955-63.

21. Suarez-Cabrera C, de la Pena B, Gonzalez LL, Page A, MartinezFernandez M, Casanova ML, Paramio JM, Rojo-Sebastian A, Moreno-Bueno G, Maroto A, Ramirez A, Navarro M. The Rasrelated gene ERAS is involved in human and murine breast cancer. Sci Rep. 2018;8(1):13038.

22. Aoyama M, Kataoka H, Kubota E, Tada T, Asai K. Resistance to chemotherapeutic agents and promotion of transforming activity mediated by embryonic stem cell-expressed Ras (ERas) signal in neuroblastoma cells. Int J Oncol. 2010;37(4):1011-6.

23. Niu Y, Jin Y, Deng SC, Deng SJ, Zhu S, Liu Y, Li X, He C, Liu ML, Zeng Z, Chen HY, Zhong JX, Ye Z, Wang CY, Zhao G. MiRNA-646-mediated reciprocal repression between HIF-1alpha and MIIP contributes to tumorigenesis of pancreatic cancer. Oncogene. 2018;37(13):1743-58.

24. Fendrich V, Jendryschek F, Beeck S, Albers M, Lauth M, Esni F, Heeger K, Dengler J, Slater EP, Holler JPN, Baier A, Bartsch DK, Waldmann J. Genetic and pharmacologic abrogation of Snail1 inhibits acinar-to-ductal metaplasia in precursor lesions of pancreatic ductal adenocarcinoma and pancreatic injury. Oncogene. 2018;37(14):1845-56.

25. Zhan HX, Xu JW, Wu D, Zhang TP, Hu SY. Pancreatic cancer stem cells: new insight into a stubborn disease. Cancer Lett. 2015;357(2):429-37.
26. Weng CC, Hawse JR, Subramaniam M, Chang VHS, Yu WCY, Hung WC, Chen LT, Cheng KH. KLF10 loss in the pancreas provokes activation of SDF-1 and induces distant metastases of pancreatic ductal adenocarcinoma in the $\operatorname{Kras}(\mathrm{G} 12 \mathrm{D}) \mathrm{p} 53$ (flox/ flox) model. Oncogene. 2017;36(39):5532-43.

27. Iwauchi T, Tanaka H, Yamazoe S, Yashiro M, Yoshii M, Kubo N, Muguruma K, Sawada T, Ohira M, Hirakawa K. Identification of HLA-A*2402-restricted epitope peptide derived from ERas oncogene expressed in human scirrhous gastric cancer. Cancer Sci. 2011;102(4):683-9.

28. Furuse J. Paradigm shifting of systemic chemotherapy for unresectable pancreatic cancer in Japan. J Clin Med. 2019;8(8):1170.

29. Park H, Bang JH, Nam AR, Eun Park J, Hua Jin M, Bang YJ, Oh DY. Prognostic implications of soluble programmed death-ligand 1 and its dynamics during chemotherapy in unresectable pancreatic cancer. Sci Rep. 2019;9(1):11131.

Publisher's Note Springer Nature remains neutral with regard to jurisdictional claims in published maps and institutional affiliations. 